\title{
Cognitive abilities in relation to frequency of seizures and neuropathology of the temporal lobes in man
}

\author{
C. B. BLAKEMORE, G. ETTLINGER, AND M. A. FAlCONER
}

From the Institute of Psychiatry and the Guy's-Maudsley Neurosurgical Unit, Maudsley Hospital, London

Several studies carried out to investigate the effects of epileptogenic discharging lesions on learning and retention reveal some uncertainty concerning the degree of behavioural disorganization produced by such lesions. The early work of Morrell, Roberts, and Jasper (1956) clearly indicated their apparent importance for conditioning. In their study conditioning to either visual, auditory, or somato-sensory stimuli was found to be impaired in monkeys with unilateral chronic epileptogenic lesions, and improvement was noted after subsequent excision of the site of the discharging lesion. One interpretation of these findings is that the unilateral discharging lesion gives rise to bilateral disturbances of cerebral function, as shown by bilateral E.E.G. abnormalities, and that the ablation of this discharging lesion restricts the functional abnormalities to the affected hemisphere, thus producing an improvement in the ease of conditioning.

Different findings are to be found in the more recent work of Stamm and his colleagues (Stamm and Pribram, 1960, 1961; Stamm and Warren, 1961) which suggest that there is less behavioural impairment following the development of discharging lesions than following ablations. They contrasted the ability of monkeys to retain tasks which had been learned before the development of bilateral discharging lesions with their ability to learn new tasks after the lesions had been established, and these findings were compared with those of similar studies using animals which had ablations of comparable areas of the cortex. They concluded that epileptogenic lesions do not impair retention for tasks once learned, but do produce an impairment of learning ability. They state that on retention tests of previously learned tasks the performance of monkeys with epileptoid discharges is essentially indistinguishable from the behaviour of normal animals. Their findings are in marked contrast with the results of ablation experiments, which show marked disruption of preoperatively learned behaviour (Stamm and Pribram, 1961). It must be noted, however, that these findings may be an artifact of the experimental procedure adopted, since the animals were repeatedly re- trained during the development of the discharging lesions, and such overlearning is known to eliminate retention defects in animals having ablations.

Relevant evidence on this subject comes from studies in which generalized convulsive seizures have been induced in animals immediately after the learning of a new task (Duncan, 1949; Heriot and Coleman, 1962; Pearlman, Sharpless, and Jarvik, 1961). Here we find that the seizure actually does impair the retention of the task, and that this impairment becomes progressively less marked with an iु increasing time interval between the end of training $\omega$ and the onset of the seizures. Relearning of the tase $N$ is not impaired by the seizure activity, but the anima $\infty$ obtains no benefit from its previous practice. Suc $\frac{1}{3}$ 을 findings would appear to lend support to the hype thesis that seizure activity interrupts in some way the consolidation of the memory process for the task. $\frac{0}{0} \mathbb{\Phi}$

Although direct experimentation in this field has $\vec{\varphi}$ been carried out with animals, studies of the effects of epilepsy in humans also provide some relevant evidence on the behavioural disorganization of cognitive abilities produced by epileptic discharges. Milner (1958) reported that her patients showed an average fall of 16 points in the Wechsler verbal I.Q. immediately after left anterior temporal lobectomy undertaken for the relief of epilepsy, but that verbal ability returned to the pre-operative level within one $\exists$ year provided there were no longer any seizures. Similarly, Blakemore and Falconer (unpublished data) have found that the length of time taken to recover auditory verbal learning ability after left $\overline{0}$ anterior temporal lobectomy is correlated with the $\dot{0}$ frequency of persisting seizures after operation. Other studies have demonstrated the differential effects on 8 verbal and perceptual tests of epileptic discharge 3 foci in various parts of the left or right hemispheres of the brain or involving mainly the dominant or non-dominant temporal lobes (Balthazar and Morrison, 1961; Dennerll, 1964; Lansdell, 1962; Meyer and Jones, 1957; Meyer, 1959; Milner, 1958). o

The present study was designed to investigate the $N$ relationship between, on the one hand, the frequency N of seizures and the severity of mesial temporal 
scleresis, a common form of epileptogenic pathology within the temporal lobes (Falconer, Serafetinides and Corsellis, 1964), and on the other hand the degree of impairment experienced by patients on standard intelligence test items. The subjects had each been given one form of the Wechsler intelligence scales before undergoing unilateral anterior temporal lobectomy, and the results of their test performance have been related to the severity of the pathological changes found at the time of operation and to the seizure frequency before operation. It may be argued that cognitive tests of the type used are not direct measures of learning and retention ability, but successful performance, particularly in respect of verbal tests, does call partly for the utilization of information and skills which the subject would have the opportunity to acquire during life. In addition, as pointed out above, such tests are known to be differentially impaired by epileptic foci in different regions of the brain, and the magnitude of these impairments may be dependent either on the severity of the neuropathological changes and/or the frequency of seizures.

\section{METHODS}

SUBJECTS The findings of the present study are based upon data obtained from 32 patients admitted to the Guy's-Maudsley Neurosurgical Unit suffering from temporal lobe epilepsy. All of the patients subsequently underwent either a left or a right anterior temporal lobectomy after the presence of an epileptic focus had been established. These cases were selected on the grounds that they satisfied each of the following criteria:

Intelligence test data The complete form I or II of the Wechsler-Bellevue intelligence scales, the Wechsler adult intelligence scale, or the Wechsler intelligence scale for children had been administered to the patient before operation. In all cases the information subtest of the verbal scale was omitted from consideration, as this is not usually administered in this Unit.

Seizure frequency The case-history material available in the records of the Unit contained sufficient information for the frequency of seizures before operation to be established.

Post-operative improvement Post-operative follow-up of the patient over a number of years indicated that seizures had ceased or their frequency was much improved after operation.

Handedness Each patient was right handed.

Pathology A detailed report was prepared previously by a particular neuropathologist (Dr. J. B. Cavanagh) on the brain tissue removed at operation, indicating the severity and extent of mesial temporal sclerosis or the presence of negligible pathology. In 1959 Dr. Cavanagh surveyed the pathological changes found in the resected temporal lobes of the first 82 patients with temporal lobe epilepsy submitted to an anterior temporal lobectomy in the Guy's-Maudsley Neurosurgical Unit, and subdivided them into three groups as follows:-
Group 1: cases with focal lesions, mostly glial hamartomas, but excluding cases in group 2 (24 cases)

Group 2: cases with diffuse and disseminated lesions including Ammon's horn sclerosis, a pathological process which was later redescribed by Falconer et al. (1964) as 'mesial temporal sclerosis' (38 cases).

Group 3: cases in which 'little or no abnormality of importance was noted', later redescribed by Falconer et al. (1964) as 'equivocal cases' (20 cases).

He further regraded all the 38 cases of 'mesial temporal sclerosis' according to the severity and extent of the changes, while he listed cases in group 3 as having negligible pathology.

Later, when this present paper was assembled, we took his pathological grading. All the 32 cases used in the present study came from the 58 cases in his groups 2 and 3 . Twenty-six cases were excluded because they did not fulfil all the other four criteria, principally because the Wechsler scores pre-operatively were not available or because the patient continued to have seizures at a rate comparable to the pre-operative level.

Data relating to the laterality of operation, age, sex, and intellectual level before operation are contained in Table I.

TABLE I

LATERALITY, AGE, SEX, AND INTELLECTUAL LEVEL BEFORE OPERATION

\begin{tabular}{|c|c|c|c|c|c|}
\hline \multirow{2}{*}{$\begin{array}{l}\text { Side of } \\
\text { Operation }\end{array}$} & \multirow[t]{2}{*}{ Age } & \multirow[t]{2}{*}{$\operatorname{Sex}$} & \multicolumn{3}{|c|}{ Wechsler Intelligence Test } \\
\hline & & & Full Scale & $\begin{array}{l}\text { Verbal } \\
\text { Scale }\end{array}$ & $\begin{array}{l}\text { Perform- } \\
\text { ance } \\
\text { Scale }\end{array}$ \\
\hline $\begin{array}{l}\text { Left } \\
(N=19) \\
\text { Right } \\
(N=13)\end{array}$ & $\begin{array}{l}\text { Mean } 25 \cdot 9 \\
\text { Range 11-40 } \\
\text { Mean 28.2 } \\
\text { Range 17-45 }\end{array}$ & $\begin{array}{r}13 \text { M } 6 \text { F } \\
8 M 5 F\end{array}$ & $\begin{array}{l}\text { Mean } 97 \cdot 79 \\
\text { Range } 125-77 \\
\text { Mean } 100 \cdot 69 \\
\text { Range } 126-75\end{array}$ & $\begin{array}{r}95 \cdot 63 \\
122-80 \\
106 \cdot 71 \\
131-75\end{array}$ & $\begin{array}{l}100 \cdot 31 \\
124-73 \\
106 \cdot 00 \\
126-78\end{array}$ \\
\hline
\end{tabular}

None of these patients had been in hospital for any considerable length of time as a result of epilepsy before admission to the Unit, and they were drawn from a wide range of occupational and socio-economic levels.

GROUPING OF SUBJECTS The 32 patients were grouped according to frequency of seizures, severity of neuropathological changes, and the laterality of hemisphere presumed to be at fault and upon which temporal lobectomy was ultimately performed.

The average number of seizures per week before operation was determined from the case history. A cut-off point was then located to divide the group into two subgroups containing 16 patients with 'maximum seizures' and 16 patients with 'minimum seizures'. The cut-off point was found to define the 'maximum' subgroup as having three or more seizures per week, while the 'minimum' subgroup had two or less seizures per week.

On the basis of the neuropathologist's report ratings of the extent and the severity of neuropathological changes were ascertained for each patient. These ratings were the average of assessments of pathology on a five-point scale for each of the following structures: the hippo- 
campus (Ammon's horn), the amygdala, the uncus, and the cortical surface of the temporal lobe. In the case of equivocal findings being reported by the neuropathologist in these structures, minimal pathology was assumed to be present; such equivocal findings were found in eight of the 32 cases. From these average ratings of neuropathology a cut-off point was located which divided the total group, again, into two subgroups of 16 patients each in terms of the degree of abnormality. These subgroups were regarded as consisting of patients with 'maximum pathology' and with 'minimum pathology', with the cut-off being situated at a rating of 1.5 on the averaged five-point scales.

ANALYSIS OF INTELLIGENCE TEST RESULTS From the divisions of the total group described above, it will be seen that each subject can be regarded as a member of each of three main subgroups: left or right hemisphere involvement, maximum or minimum frequency of seizures, and maximum or minimum severity of pathology. The analysis of the intelligence test results was based on comparisons of the scores of patients falling into each of the possible combinations of these eight subgroupings. The actual number of subjects in each of the possible categories is shown in Table II.

\section{TABLE II}

GROUPING OF PATIENTS BY LATERALITY OF HEMISPHERE INVOLVED, FREQUENCY OF SEIZURES, AND SEVERITY OF NEUROPATHOLOGY

\begin{tabular}{lcccc} 
Hemisphere & $\begin{array}{l}\text { Maximum } \\
\text { Seizures } \\
\text { Maximum } \\
\text { Pathology }\end{array}$ & $\begin{array}{l}\text { Minimum } \\
\text { Seizures } \\
\text { Maximum } \\
\text { Pathology }\end{array}$ & $\begin{array}{l}\text { Maximum } \\
\text { Seizures } \\
\text { Minimum } \\
\text { Pathology }\end{array}$ & $\begin{array}{l}\text { Minimum } \\
\text { Seizures } \\
\text { Minimum } \\
\text { Pathology }\end{array}$ \\
\hline Left $(\mathbf{N}=19)$ & 6 & 4 & 6 & 3 \\
Right $(\mathbf{N}=13)$ & 2 & 4 & 3 & 4 \\
Total $(\mathbf{N}=32)$ & 8 & 8 & 9 & 7
\end{tabular}

The weighted scores, with corrections for age where necessary, of the 10 subtests of the Wechsler scales, $Z$ together with full-scale, verbal-scale, and performance scale total weighted scores were each analysed separatelyo by an analysis of variance. This analysis comparede differences for each set of scores due to the principalo effects of seizure and pathology groupings and of hemi- 0 sphere involvement, and also examined the possible significance of interactions between hemisphere involve- $=$. ment and the other groupings. The procedure adopted for? each of the analyses was that recommended for a factoriak experiment with unequal cell frequencies by Winer (1962) $\overrightarrow{\vec{F}}$

\section{RESULTS}

The findings of this investigation are shown in Table III. Here the results of the analyses of data from each of the intelligence test scales and subtests ares shown in terms of mean scores and the level of significance of differences between the various groupings.

It will be seen that none of the differences between the major groupings for the frequency of seizures and the severity of neuropathology reach an acceptable level of significance in the individual analyses of variance for the intelligence test variables. Whifle none of these differences are statistically significant ${ }_{\infty}^{\circ}$ it is worth noting that in the case of all five verfab subtests the lowest mean score is always associated with the 'maximum seizures' groupings, irrespectise of the severity of pathology. The total weighted sceres for the verbal scale reflects this to some extent, ${ }^{\mathbb{D}}$ although the cumulative effect of these subtesto differences is, again, not statistically significant an acceptable level (falling just short of the $p=0.05$

TABLE III

MEAN SCORES ON WECHSLER INTELLIGENCE TEST SCALES AND SUBTESTS AND LEVEL OF SIGNIFICANCE FOR COMPARISONS욤 BETWEEN GROUPS

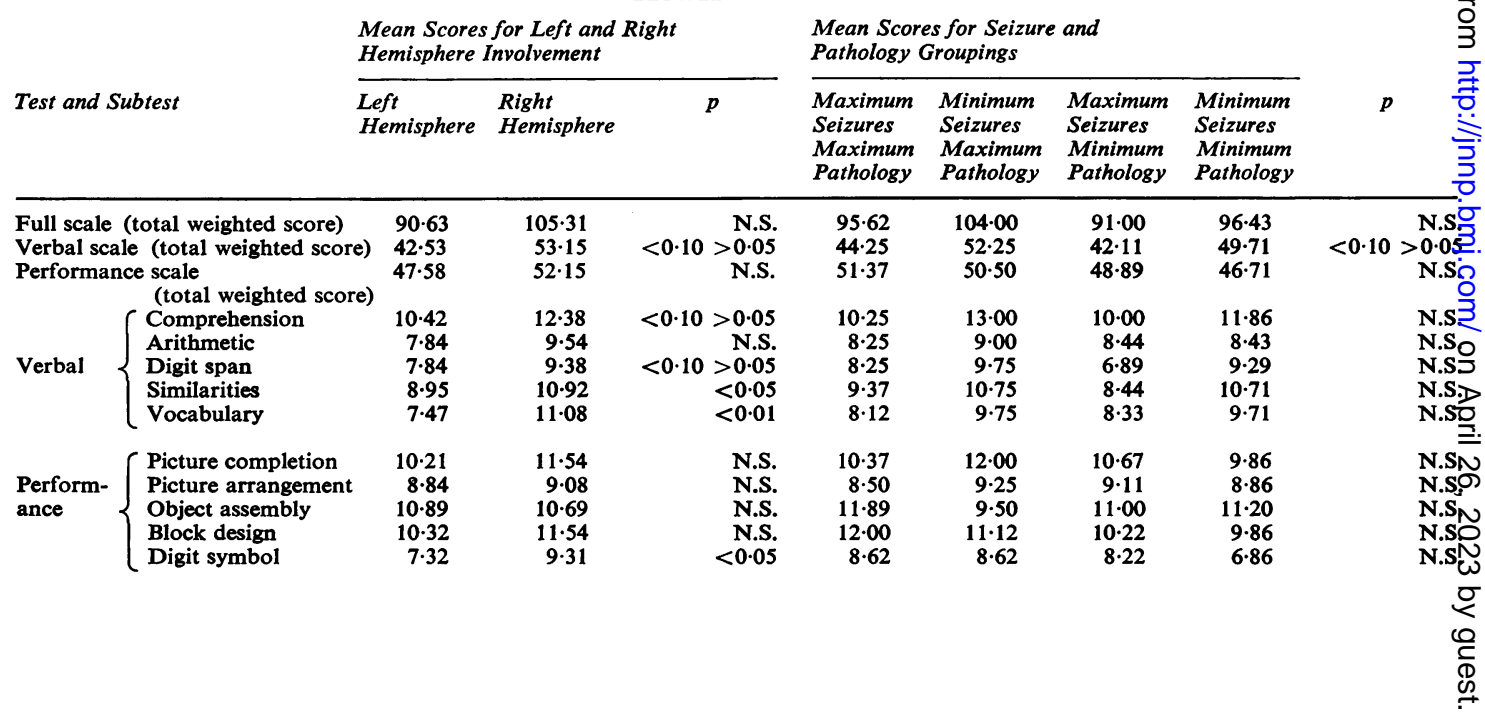


level). No such clear pattern of differences appeared to emerge in the case of the performance subtests.

When the results of the analysis by laterality of temporal lobe involvement are considered, we find statistically significant differences in terms of poorer performance on the similarities, vocabulary, and digit symbol subtests by the left hemisphere group. There is also a tendency, although not reaching a high level of statistical significance, for the left hemisphere group to be impaired on tests of comprehension and digit span, and also on the verbal scale total weighted score.

From the analyses of variance it was found that with none of the scales or subtests was there a significant interaction between laterality of temporal lobe involvement and the various groups for seizure frequency and severity of neuropathological changes.

\section{DISCUSSION}

While our findings are generally inconclusive in relation to the effects on cognitive behaviour of epileptic lesions, it must be remembered that the numbers in each of our groups and subgroupings were small. With larger groups certain of the tendencies observed, but which here fall short of an acceptable level of statistical significance, might have been more clearly revealed. It is also possible that more positive findings might have been obtained if our analysis had taken account of additional variables. One such variable which we would have wished to include in this study is the age of onset of seizures, by grouping patients with onset of seizures before and after 10 years of age, but unfortunately our total number of patients was too small to permit an adequate analysis of the data if this additional variable had been included. This may be a relevant variable, however, for the distribution in terms of our seizure frequency and severity of pathology groupings suggests that patients whose seizures begin before 10 years of age tend to fall mainly in the 'maximum seizures/maximum pathology' or 'minimum seizures/minimum pathology' groupings, while with seizures starting after 10 years they tend to fall mainly in the 'maximum seizures/minimum pathology' or 'minimum seizures/maximum pathology' groupings.

The conclusions to be drawn from this study would seem to be that cognitive abilities have not been shown to be significantly related to the severity of epileptic lesions in the temporal lobe of humans. There is, however, some suggestion from the analysis of hemisphere laterality that the deficits observed in our cases are not dissimilar from those found after excision of one anterior temporal lobe, with the most marked effect being a general impairment on tests of verbal intelligence and verbal retention with left temporal involvement (Meyer, 1959; Milner, 1958). In keeping with this is our finding that ability in the similarities subtest is more impaired in the case of left rather than right temporal lobe involvement, which may extend Reitan's (1957) proposition that ability in this subtest is always severely impaired in cases of temporal lobe pathology. We must also bear in mind the observation of the tendency for impairment of verbal abilities to occur in relation to the frequency of seizures, rather than in relation to the severity of neuropathology. If we look upon verbal intelligence test items as reflecting to some extent the ability to store and utilize concepts and information acquired during life, this observation may lend some support to the notion that the greater the epileptic disturbance caused by a lesion the greater the degree of cognitive disorganization.

We offer these findings to demonstrate how data from neurological and neurosurgical patients can be analysed in relation to a problem such as the effects of epileptogenic lesions on cognitive behaviour, and in the hope that they may stimulate other workers to improve upon this approach in future studies.

\section{SUMMARY}

The pre-operative performance on standard intelligence test items of 32 patients submitted to anterior temporal lobectomy for the relief of epilepsy was analysed in relation to frequency of seizures before operation and severity of neuropathological changes found at the time of operation. These patients were grouped according to laterality of temporal lobe involvement, and were divided into subgroups for maximum and minimum frequency of seizures and maximum and minimum severity of pathology. The findings in relation to the effects on cognitive behaviour of laterality of involvement are in general agreement with previous studies. While the findings are inconclusive in relation to the effects of epileptic lesions on cognitive behaviour, there is some evidence, although statistically not highly significant, to suggest that impairment on verbal intelligence test items is more dependent upon frequency of seizures than upon severity of neuropathological changes.

We wish to thank Dr. A. E. Maxwell, Statistical Unit, Institute of Psychiatry, for his advice on the statistical analysis of our data. The authors also wish to acknowledge the assistance they received from having access to the neuropathological grading of patients prepared by Dr. J. B. Cavanagh, now at the Institute of Neurology, National Hospital, Queen Square, London, W.C.1. 


\section{REFERENCES}

Balthazar, E. E., and Morrison, D. H. (1961). The use of Wechsler Intelligence Scales as diagnostic indicators of predominantly left-right and indeterminate unilateral brain damage. J. clin. Psychol., 17, 161-165.

Dennerll, R. D. (1964). Cognitive deficits and lateral brain dysfunction in temporal lobe epilepsy. Epilepsia (Amst.), 4 ser., 5, 177-191.

Duncan, C. P. (1949). The retroactive effect of electroshock on learning. J. comp. physiol. Psychol., 42, 32-44.

Falconer, M. A., Serafetinides, E. A., and Corsellis, J. A. N. (1964). Etiology and pathogenisis of temporal lobe epilepsy. Arch. Neurol. (Chic.), 10, 233-248.

Heriot, J. T., and Coleman, P. D. (1962). The effect of electroconvulsive shock on retention of a modified 'one-trial' conditioned avoidance. J. comp. physiol. Psychol., 55, 1082-84.

Lansdell, H. (1962). Laterality of verbal intelligence in the brain. Science, 135, 922-923.

Meyer, V. (1959). Cognitive changes following temporal lobectomy for relief of temporal lobe epilepsy. Arch. Neurol. Psychiat. (Chic.), 81, 299-309.

_, and Jones, H. G. (1957). Patterns of cognitive test performance as functions of the lateral localization of cerebral abnormalities in the temporal lobe. J. ment. Sci., 103, 758-772.
Milner, B. (1958). Psychological defects produced by temporal obe $\rho$ excision. In The Brain and Human Behavior. Williams and

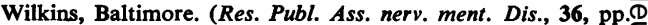
244-257).

Morrell, F., Roberts, L., and Jasper, H. H. (1956). Effect of focap epileptogenic lesions and their ablation upon conditioned electrical responses of the brain in the monkey. Electroencepho clin. Neurophysiol., 8, 217-236.

Pearlman, C. A., Sharpless, S. K., and Jarvik, M. E. (1961). Retro-n grade amnesia produced by anaesthetic and convulsant agents. J. comp. physiol. Psychol., 54, 109-112.

Reitan, R. M. (1957). Differential patterns of psychological testō results in lateralized and localized cerebral lesions. Proc. $X V$ th international Congress of Psychology. Brussels, Belgium, 1957.

Stamm, J. S., and Pribram, K. H. (1960). Effects of epileptogenion lesions in frontal cortex on learning and retention in monkeys. J. Neurophysiol., 23, 552-563.

- and - (1961). Effects of epileptogenic lesions of inferotemporal cortex on learning and retention in monkeys. J. comp $\overline{\bar{s}}$ physiol. Psychol., 54, 614-618.

- and Warren, A. (1961). Learning and retention by monkeys with epileptogenic implants in posterior parietal cortex Epilepsia (Amst.), 4 ser., 2, 229-242.

Winer, B. T. (1962). Statistical Principles in Experimental Design,page 241. McGraw-Hill, New York. 